\title{
Timed Trajectory Generation Combined with an Extended Kalman Filter for a Vision-Based Autonomous Mobile Robot
}

Jorge B. Silva, Cristina P. Santos and João Sequeira

\begin{abstract}
Planning collision-free trajectories requires the combination of generation and modulation techniques. This is especially important if temporal stabilization of the generated trajectories is considered. Temporal stabilization means to conform to the planned movement time, in spite of environmental conditions or perturbations. This timing problem has not been addressed in most current robotic systems, and it is critical in several robotic tasks such as sequentially structured actions or humanrobot interaction. This work focuses on generating trajectories for a mobile robot, whose goal is to reach a target within a constant time, independently of the world complexity. Trajectories are generated by nonlinear dynamical systems. Herein, we extend our previous work by including an Extended Kalman Filter (EKF) to estimate the target location relative to the robot. A simulated hospital environment and a Pioneer 3-AT robot are used to demonstrate the robustness and reliability of the proposed approach in cluttered, dynamic and uncontrolled scenarios. Multiple experiments confirm that the inclusion of the EKF preserves the timing properties of the overall architecture.
\end{abstract}

\section{Introduction}

The ability to plan collision-free trajectories for autonomous mobile robots includes fundamental issues, such as trajectory modulation and generation. This issue becomes more relevant in the path planning problem if temporal stabilization of the generated movements is considered, particularly in discrete movements.

Jorge B. Silva

University of Minho, Guimarães, Portugal, e-mail: jbruno@ dei.uminho.pt

Cristina P. Santos

University of Minho, Guimarães, Portugal e-mail: cristina@dei.uminho.pt

João Sequeira

Instituto Superior Técnico, Lisbon, Portugal e-mail: jseq@irs.ist.utl.pt 
Temporal stabilization means to conform to the planned movement time, despite varying environmental conditions or perturbations. Thus, robot movements must compensate for disturbances, accelerating or decelerating. This timing problem has not been addressed in most current robotic systems. For instance, the classical problem of autonomous navigation, tasks performed by the robot only require a convenient overall speed and when a robot takes longer to arrive at a goal because of some unforeseen disturbance such as obstacle circumnavigation, this change of timing is not compensated for by accelerating the vehicle along its trajectory.

Timed actions, involve stable temporal relationships and this temporal stabilization is critical in multiple robotic tasks including avoidance of moving objects, catching, hitting, drumming, juggling, coordination between degrees of freedom, human-robot scenarios and generating sequentially structured actions.

In this contribution we continue previous work attempts to tackle these spatialtemporal problems. Herein, we extend our previous work aiming that the robot reaches a goal location while avoiding obstacles within a specified movement time [1]. However, the target which identifies the goal location was defined geographically. To make the application more realistic, in [2], the sensory loop was closed by online acquiring the location of the target through the built-in robot's visual system, such that action was steered by sensory information. However, when the localization of a target is obtained from a vision system there may be situations in which the robot's visual system is unable to detect the respective target. Obstacles in the environment occluding the target are an example of such situations.

An Extended Kalman Filter (EKF) is used to estimate the location of a ball relative to the current position of the mobile robot. We have chosen to apply an EKF because this filter has a low computational cost, and it is an optimal estimator that linearizes the nonlinear system around the estimation. Note that our focus is to develop an architecture that integrates the dynamical systems theory and an EKF while providing for the timed control of an autonomous mobile robot. The resulting architecture is useful in robotics applications that have to generate rhythmic movements and simultaneously to deal with targets, which can be visible or undetectable during periods of time.

However, an important question which has to be considered is whether the inclusion of an EKF affects the temporal nature of the previously proposed dynamical system architecture, i.e., the robot has to keep reaching the target location within the specified time.

Comparative examples of temporal solutions with and without the EKF are presented which show the intrinsic robustness of the system even in the presence of non-modeled dynamics (Brownian motion of the target).

The proposed architecture is verified in a hospital environment. In such application, the robot is expected to carry out a task or a sequence of tasks within a specified timing. Specifically, if we consider tasks that require a human-robot interaction, or if the mobile robot has to delivery or carry goods between different locations of the hospital. 
In such case, it is required to guarantee that the response time will be inferior to a certain value, which depends on the type of interaction, and which can be specified by higher levels of the modular architecture herein presented.

The experiments were performed in a Webots simulation environment using a Pioneer 3-AT robot. The EKF was able to robustly estimate the location of the target even when the path covered by the robot is subjected to variations not known a priori, and to converge to the reference path after long periods of time without observations. This is especially important if the robot has to navigate in cluttered environments.

\section{Related Work}

The generation of timed trajectories embedded in feedback structures remains, to the best of our knowledge, an issue currently receiving attention both in robotics and in neurosciences. Usually the proposed control approaches are based on nonlinear dynamical systems.

Research has been revolving around related themes considering learning by demonstration, including the dynamical motor primitives [3], combining dynamics for rhythmic and discrete motion, generation of synchronized movement for robot with multiple degrees of freedom to achieve tasks which involve rhythmic motions such as biped and quadruped autonomous adaptive locomotion over irregular terrain [4], juggling [5], drumming [3], playing with a slinky toy, basis field approaches for limb movements [6] and locomotion [7] for instance, as well as superposing movement primitives.

The proposed systems are robust against disturbances but temporal stabilization is not effectively attempted. These ideas were previously extended to signal processing in biped and quadruped locomotion [8], using a frequency-learning mechanism for nonlinear oscillators in the presence of external signals.

In [9] the drumming problem is also addressed but it does not take into account any kind of disturbance which may affect the rhythmic movement. Also [10], combined rhythmic and discrete movements were able to generate movements in a BallPaddling robotic application.

Human-like reaching has been implemented in a robot manipulator [11] to reach a tracked ball by applying VITE-like dynamical systems. Recent studies include robust manipulation through the learning of motion primitive goal [12].

Nevertheless, works addressing the generation of rhythmic movements in wheeled mobile robots has not been addressed in most current works. In [13], rhythmic movement is generated using limit cycle attractors for a wheeled mobile robot that is able to perform a sequence of basic behaviors. However, an inherent limitation of this approach is that it only generates a single continuing pattern of rhythmic movement, remaining still limited with respect to the integration of multiple constraints and discrete movements. 
These works have used nonlinear dynamical systems, including oscillators, and the oscillatory behavior has been explored for responsiveness and flexibility as well as for timing. The dynamical systems theory provides several advantages to address the temporal stabilization problem such as low computational cost, smooth online modulation while keeping the general features of the original movements, robustness against small perturbations, and possibility to integrate sensory feedback.

The work in [14] has initiated the dynamical system approach to understand movement coordination to generate timed actions, in which time is a controlled variable against perturbations when a particular action is initiated and terminated. In [15], this architecture was implemented in a real vehicle, where a temporal stabilization mechanism was proposed. The same framework was modified in [16], in which some changes to the approach were proposed, since they argued that the included temporal stabilization mechanism was not able to preserve the temporal constraint.

We continued to tackle this challenge and proposed a few novel adaptations to the system in [1], by exploring the intrinsic properties of the used oscillator. The main novelty was in exploring bifurcation theory to switch the qualitative dynamics of the oscillators, instead of switching among different dynamical systems. Thus, we generated sequences of both discrete and rhythmic movements through the same system similarly to work in locomotion [17] and [4], but applied to a wheeled robot. Further, an adaptive mechanism for frequency modulation of the velocity profile based on the oscillators current state was proposed, allowing to set different times for acceleration and deceleration.

The applied type of movements, discrete and rhythmic, is strongly supported by research on human motor control [18].

In mobile robotics the EKF has been extensively used e.g., to smooth data from ultrasonic sensors in order to estimate the location of objects [19] or in robotic arms catching a flying ball [20],[21] where a ball is launched towards to the robotic arm, and an EKF is used to track and to predict the position of the ball at each instant of time. EKFs have been also widely spread in the literature addressing target tracking. However, its effects in the overall dynamics have not received much attention, e.g., related to target occlusion during large periods of time. This is an aspect enforced by the experiments presented here.

\section{System Overview}

The overall architecture of our system is depicted in fig 1 . The system is hierarchically divided in two functional layers according to their level of abstraction. These two layers implemented through a mesh of interacting dynamical systems, are mutually coupled, however they work independently of one another, and each layer sends sets of parameters to the other layer. The second layer mimics the role of supra-spinal structures in biological systems. Basically, it is responsible for selecting the most adequate robot motor behavior accordingly to external conditions and 


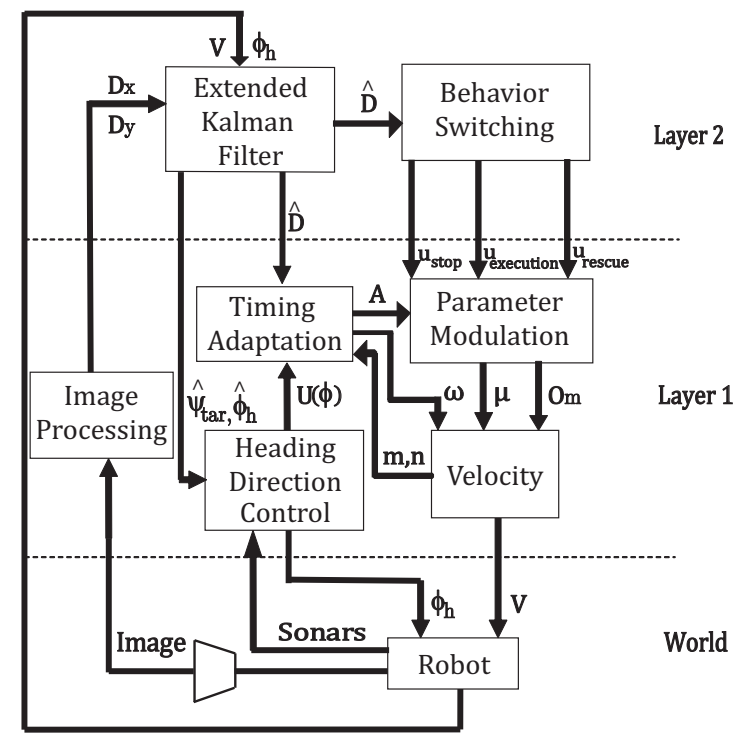

Fig. 1 System overall schematic.

current states of the task at hand, map it onto the corresponding set of dynamical parameters and send them to the first layer at the right timing.

The first layer focuses its operation on controlling the 2D motion of the robot according to the dynamics of two behavioral variables: the heading direction $\phi_{h}$ and the forward velocity $v$.

In the following, we detail each layer of the overall architecture. For a complete explanation please see $[2,1]$.

\subsection{First Layer}

For heading control, we motivate the choice of the dynamical systems approach [22], because its properties allow for the integration of sensory-motor feedback and thus for closed-loop control. The directional dynamics rules the robot's heading direction $\phi_{\mathrm{h}}$ by avoiding obstacles and following towards to the target.

The velocity $v$ of the robot is the major determinant to the success of the movement task within the specified timing constraints. Velocity is set as the solution of a stable limit-cycle generated by a Landau-Stuart oscillator $[2,15,1]$, which is modulated by the parameter modulation. The performed velocity consists of a single oscillation cycle, adapted in order to accelerate or decelerate in the presence of disturbances. 
The proposed oscillator is able to generate discrete, rhythmic or a superimposition of both movements, resulting in a more complex movement. The choice and switch among these different movements depends explicitly on the parameters values.

Timing adaptation is achieved by computing online an updating rule for the oscillator parameters. It is explained detail in [2] as well as obstacle avoidance.

This layer receives from the second layer at the right timing, sets of parameters that specify and modulate in a simple and straightforward manner the generated trajectories.

\subsection{Second Layer}

Commands are sent from the second layer, changing qualitatively the dynamic solution by taking advantage of the Hopf bifurcation of the Landau-Stuart oscillator, resulting in three motor behaviors, available in the level's repertoire: stop, the robot does not move; execution, the robot performs the rhythmic movement; rescue, discrete movement.

The Behavior Switching module autonomously selects and sequences these motor behaviors accordingly to external conditions and current states of the task at hand, such that action itself is elicited by perception.

The Extended Kalman filter module, employs an EKF in order to estimate the localization of the target relatively to the robot's position, i.e. the $\hat{D}_{x}, \hat{D}_{y}$ projections onto the robot $\mathrm{X}$ and Y-axes of the remaining distance to reach the target, and consequently the distance $\hat{D}$ to the target and the direction $\hat{\psi}_{\text {tar }}$ where the target lies ${ }^{1}$.

It receives from the Image processing module the measured $D_{x}$ and $D_{y}$.

Note that the timing adaptation is directly proportional to the estimation of $\hat{D}$ and thus the EKF has a very important role in the overall architecture.

\subsection{Timing Constraints}

This timing architecture is very useful for robotic applications that need to generate robust timed movements. Moreover, the architecture is able to generate rhythmic movements that meet the requirements imposed by the dynamical parameters. This means that the proposed dynamics are to a large extent independent from the physical implementation. The necessary modifications are only required at the level of the heading direction dynamics.

However, these timing movements are bounded due to the robot physical constraints. Herein, we can identify physical limitations such as accelerations or decelerations of the robot or even the maximum velocity reached by the robot. Let us

\footnotetext{
${ }^{1}$ Along the paper this robot frame refers to a reference frame that has origin located at the position of the robot and is always aligned with an inertial world frame.
} 


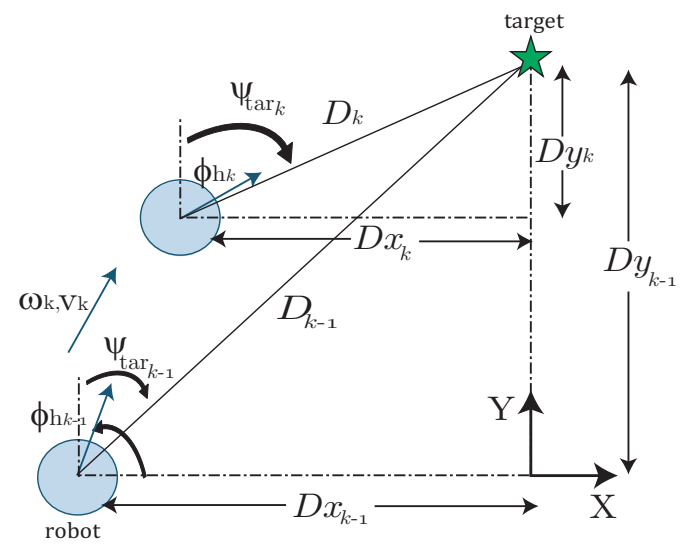

Fig. 2 Representation of the variables involved in the motion of the robot relative to the target. Variables are described in the robot coordinate system. The robot has linear and angular velocity in both instants of time.

consider a complex and cluttered environment, for instance a hospital environment, in which a mobile robot has to perform timed sequentially delivery tasks. The timing movements are constrained by the maximum velocity that the robot can reach in such environments. This is dependent on the velocity that the users move in the environment, in the degree of clustering and also on its own achievable velocity. This is a typical physical limitation of the robotic application and not from the proposed timing architecture.

\section{Extended Kalman Filter Formulation}

The EKF needs a state space model of the dynamic system describing the time evolution of the state to be estimated, herein the target position in the robot coordinate system, expressed by the system state discrete variable $x_{k}=\left[D x_{k}, D y_{k}, \phi_{\mathrm{h}_{k}}\right]^{T}$.

In order to calculate the localization of a target, we need to calculate the distance $D$ between the robot and the target, and the angle $\psi_{\text {tar }}$ from the robot X-axes to the target around the robot Z-axes. Figure 2 details the variables involved when representing the motion of the robot relative to the target. All variables are defined in the robot coordinate system.

The following relationships may be established among these variables:

$$
\begin{aligned}
D x_{k} & =D x_{k-1}-v_{k} \cos \left(\phi_{\mathrm{h}_{k}}\right) \mathrm{dt}, \\
D y_{k} & =D y_{k-1}-v_{k} \sin \left(\phi_{\mathrm{h}_{k}}\right) \mathrm{dt}, \\
\phi_{\mathrm{h}_{k}} & =\phi_{\mathrm{h}_{k-1}}+\omega_{k} \mathrm{dt},
\end{aligned}
$$


where $D x_{k}, D y_{k}$ are the projections onto the robot $\mathrm{X}$ and Y-axes of the distance between the robot and the target, $\phi_{\mathrm{h}_{k}}$ is the current heading direction of the robot, $v_{k}$ is the robot forward velocity and $\omega_{k}$ is the robot angular speed.

The angle that the robot has to turn in order to face the target $\psi_{\mathrm{tar}_{k}}$ and the distance $D_{k}$ that the robot has to travel to reach the target at instant $k$ are given by:

$$
\begin{aligned}
\psi_{\operatorname{tar}_{k}} & =\arctan \left(\frac{D x_{k}}{D y_{k}}\right), \\
D_{k} & =\sqrt{D x_{k}^{2}+D y_{k}^{2}} .
\end{aligned}
$$

A nonlinear system describing equations 1,2,3 can be expressed as follows:

$$
\begin{aligned}
& x_{k}=f\left(x_{k-1}, u_{k-1}\right)+w_{k}, \\
& y_{k}=h\left(x_{k}\right)+z_{k},
\end{aligned}
$$

where $u_{k-1}=\left[v_{k}, \omega_{k}\right]^{T}$ are the control variables.

The random variables $w_{k}$ and $z_{k}$ represent the process and measurement noise respectively. They are assumed to be white and independent of each other, and with normal probability distributions.

Let us write the new governing equations that linearize an estimate about eqs. 6 and 7:

$$
\begin{aligned}
x_{k} & \approx \hat{x}_{k}^{-}+F_{k}\left(x_{k-1}-\hat{x}_{k-1}\right)+W_{k} w_{k-1}, \\
y_{k} & \approx \hat{y}_{k}^{-}+H_{k}\left(x_{k-1}-\hat{x}_{k-1}\right)+Z_{k} z_{k-1},
\end{aligned}
$$

where $F_{k}$ and $H_{k}$ are the Jacobian matrices of partial derivatives of $f$ and $h$ with respect to $x . W_{k}$ and $Z_{k}$ are the Jacobian matrices of partial derivatives of $f$ and $h$ with respect to $w$ and $z$ respectively.

The EKF time update equations are:

$$
\begin{aligned}
\hat{x}_{k}^{-} & =f\left(D x_{k-1}, D y_{k-1}, \phi_{\mathrm{h}_{k-1}, u_{k-1}}\right) \\
P_{k}^{-} & =F_{k} P_{k-1} F_{k}^{T}+Q,
\end{aligned}
$$

where $P_{k}^{-}$is the a priori error covariance, $P_{k}$ is the a posteriori error covariance, and $\hat{x}_{k}^{-}$is the state estimated a priori. The EKF measurement update equations:

$$
\begin{aligned}
K_{k} & =P_{k}^{-} H_{k}^{T}\left(H_{k} P_{k}^{-} H_{k}^{T}+R\right)^{-1} \\
N_{k} & =y_{k}-h\left(\hat{x}_{k}^{-}\right)-y_{k}-h\left(\hat{x}_{k}^{-}\right) \\
\hat{x}_{k} & =\hat{x}_{k}^{-}+K_{k} N_{k} \\
P_{k} & =\left(I-K_{k} H_{k}\right) P_{k}^{-}
\end{aligned}
$$

where $K_{k}$ is the Kalman gain, $N_{k}$ is the innovation, and $\hat{x}_{k}$ represents the state estimated a posteriori. The initial error covariance $P_{0}$ is set equal to $R$. 
$R$ was obtained through some off-line samples of the measurements $\left(D x_{k}, D y_{k}\right.$, $\phi_{\mathrm{h}_{k}}$ ), from which the respective covariance was computed.

\section{Performance of the Extended Kalman Filter}

In order to analyze the impact of the EKF in the timing properties of the architecture and on the planned movement time, comparative examples of the robot moving towards a target with and without the EKF are evaluated.

In these experiments the robot has to reach a target at a distance of $4 \mathrm{~m}$ within $30 \mathrm{~s}$, no obstacles appear during its direct path and the target is always visible to the robot's vision system. Table 1 shows the obtained results for several performance indexes defined ahead. The target position is perceived by the robot's visual system as if the target is moving inside an hypothetical circumference, whose radius is varied in the different experiments.

Due to the robot's physical constraints, we consider that the noise in the target position is perceived only during the first $27 \mathrm{~s}$ of each experiment. After this instant of time the target will remain in the same position in order for the robot to have enough time to reach it.

The value $\Delta \mathrm{P}_{f}$ indicates the Euclidean distance between the target position and the final position of the robot. $\Delta \mathrm{P}$ is the Euclidean distance between the robot and the target at $t=27 \mathrm{~s}$. In the same experiment without noise, the value of $\Delta \mathrm{P}$ is $0.197 \mathrm{~m} . \Delta \mathrm{T}$ indicates if the time constraints were verified. It is calculated as the ratio between the time needed by the robot to reach the target and the specified movement time. If this value is smaller than 1 the timing properties are verified.

Table 1 Performance of the system resulting from different perceptions of the target noise.

\begin{tabular}{|c|c|c|c|c|c|}
\hline Radius & & $\Delta \mathrm{P}(\mathrm{m})$ & $\Delta \mathrm{P}_{f}(m)$ & $\Delta \mathrm{T}(\mathrm{s})$ & $\mathrm{E}$ \\
\hline $5 \mathrm{~cm}$ & without Kalman & 0.222 & 0.034 & 0.970 & 0.1155 \\
\hline $5 \mathrm{~cm}$ & with Kalman & 0.181 & 0.023 & 0.957 & 0.1148 \\
\hline \hline $10 \mathrm{~cm}$ & without Kalman & 0.231 & 0.036 & 0.966 & 0.1163 \\
\hline $10 \mathrm{~cm}$ & with Kalman & 0.185 & 0.011 & 0.93 & 0.1140 \\
\hline \hline $20 \mathrm{~cm}$ & without Kalman & 0.195 & 0.039 & 0.960 & 0.1166 \\
\hline $20 \mathrm{~cm}$ & with Kalman & 0.196 & 0.007 & 0.921 & 0.1104 \\
\hline \hline $30 \mathrm{~cm}$ & without Kalman & 0.184 & 0.040 & 0.957 & 0.1166 \\
\hline $30 \mathrm{~cm}$ & with Kalman & 0.189 & 0.031 & 0.939 & 0.1105 \\
\hline
\end{tabular}

From table 1, when the EKF is used the error in the final position of the robot, $\Delta \mathrm{P}_{f}$, is smaller. Regarding $\Delta \mathrm{P}$ also we can verify that when the noise perception is disabled, at $t=27 \mathrm{~s}$, the difference between this value and the ideal $\Delta \mathrm{P}=0.197$ is smaller when the EKF is used.

Also, it can be verified that $\Delta \mathrm{T}$ is smaller than 1 . Therefore, the inclusion of the EKF besides reducing the error in the target perception did not compromise the timing properties. 
The amount of energy required by the robot to move is estimated as $E=$ $\sqrt{v^{2}+\omega^{2}}$. These results clearly show that the average energy required for the robot to successfully complete the missions is lower when the EKF is used.

\section{Experimental Results}

Here we describe some experiments realized in a simulated hospital environment using a Pioneer 3-AT robot. The robot has no previous knowledge about the position of any obstacle in the world, or other disturbance that possibly may occur. If the robot stops detecting the ball by any reason, the EKF keeps providing at each instant of time the direction to the ball, as well as the current distance between the ball and the robot.

At each sensorial cycle, information is acquired, dynamic equations are calculated and integrated using an Euler method with a time step of $200 \mathrm{~ms}$. The robot is able to reach a maximum velocity of approximately $0.75 \mathrm{~m} / \mathrm{s}$.

In all the presented experiments the robot always looses visual contact with the ball when the distance between both is small $(\approx 0.4 \mathrm{~m})$, because the ball's size is larger than the camera's field of view and the visual system of the robot is automatically disabled.

\subsection{Experiment 1}

This first experiment aims to demonstrate the EKF performance when the robot is compensating the disturbance caused by two obstacles that force an obstacle circumnavigation and to cover more distance (fig. 3). Thus, the robot has to increase its velocity in order to compensate for this delay. Additionally, when the robot is overcoming the obstacles it looses visual contact with the ball.

Fig. 4 shows the $D_{x}$ (top) and $D_{y}$ (middle) measured (green continuous line) and estimated (black dashed line). The bottom panel depicts the estimated $\hat{\phi}_{\mathrm{h}}$, the measured robot's heading direction $\phi_{\mathrm{h}}$, and the angle $\hat{\psi}_{\mathrm{tar}}$, provided by eq 4 . Shadow areas represent intervals of time when the robot does not detect the target, $8 s \leq t<31 s$ due to the obstacle circumnavigation and after $t>45 s$ due to the target proximity. The EKF keeps providing good estimates even after the long period without observations, showing a fast convergence to the real value, despite the time spent without new sensorial information. This is a specially important behavior if the robot has to navigate in cluttered environments. 


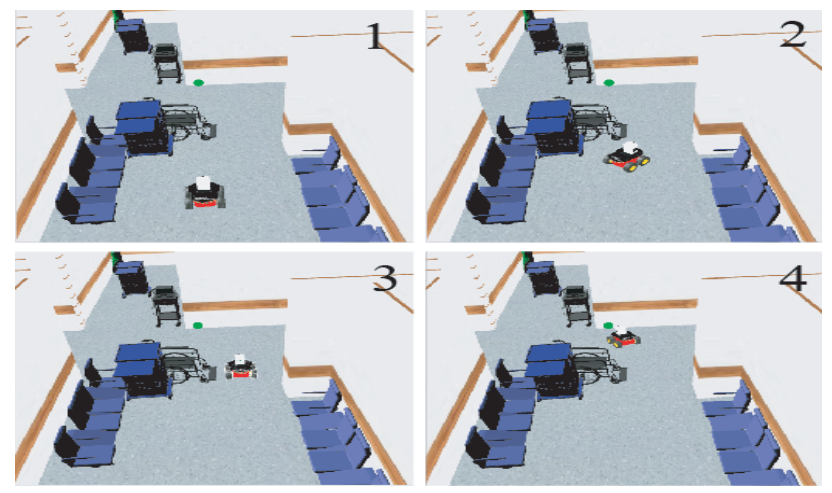

Fig. 3 Snapshots of robot motion when it is moving towards to the ball and has to compensate for the disturbance created by obstacles.
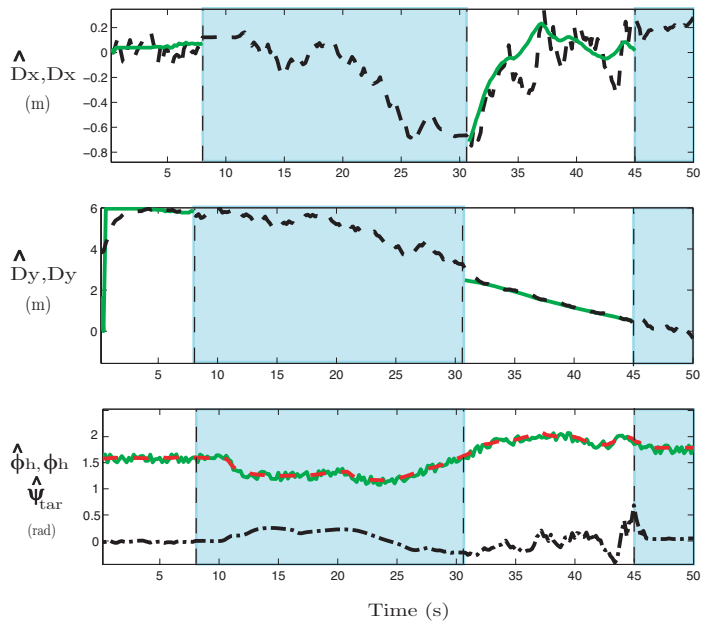

Fig. 4 Top panel (Middle): Measured $D_{x}\left(D_{y}\right)$ by the robot's visual system (green continuous line), and estimated $\hat{D}_{x}\left(\hat{D}_{y}\right)$ by the EKF (black dashed line). Bottom: Estimated robot's heading direction $\hat{\phi}_{\mathrm{h}}$ (dashed red line), the one calculated by the dead-reckoning $\phi_{\mathrm{h}}$ (continuous green line), and the angle $\hat{\psi_{\text {tar }}}$ (continuous-dashed black line) that the robot has to update its heading direction in order to reach the target.

\subsection{Experiment 2}

In this experiment we want to verify the EKF behavior when the target introduces non-modeled dynamics. Initially the distance between the robot and the ball is approximately $4 \mathrm{~m}$, and the robot starts moving towards to it. However, as the robot is moving the position of the ball is disturbed. 

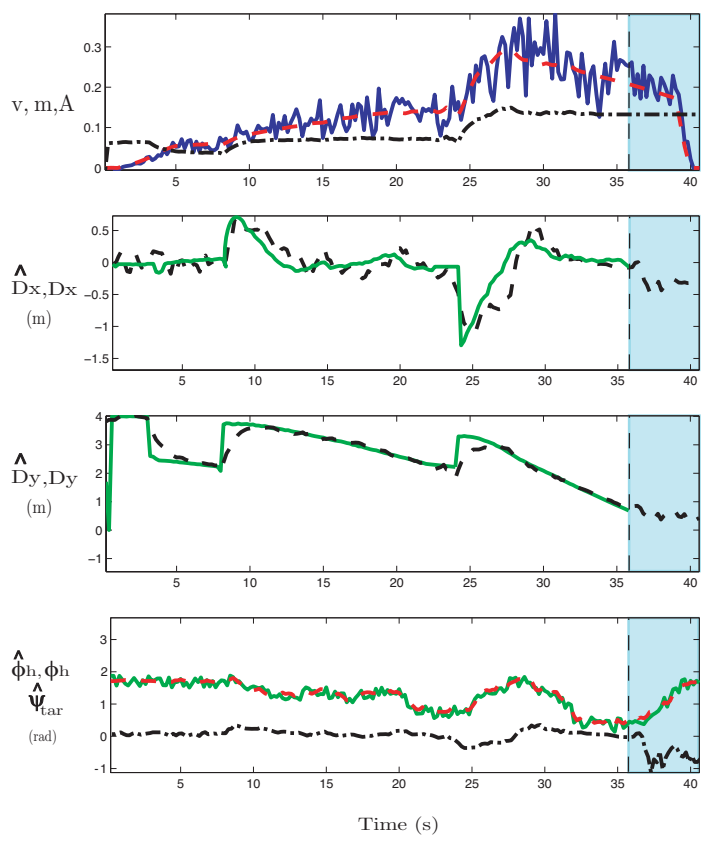

Fig. 5 Similar to fig. 4 but for the experiment 2.

The velocity profile is presented in the first panel of fig. 5. Blue continuous line identifies the measured robot's velocity through odometry, red dashed line depicts the generated velocity by the oscillator and the continuous-dashed black lines describes the amplitude of the respective oscillator. The second, third and bottom panels depict the same variables identified in fig. 4 . At $t=3 \mathrm{~s}$, when the target moves the amplitude of the oscillator is decreased according and consequently decreased the robot's velocity. At $t=8 \mathrm{~s}$ and at $t=25 \mathrm{~s}$, a new displacement of the target happens and a new adjustment of the robot's velocity is required. With these adjustments the robot can reach the target within the specified movement time.

\subsection{Experiment 3}

In the final experiment we aim to verify if the noise resultant from non-modeled dynamics can be removed by the EKF. The ball presents a Brownian movement.

In fig. 6 we can verify that the EKF is able to cope with the unmodeled Brownian motion dynamics of the target within the required $30 \mathrm{~s}$. These experiments were performed for a noise standard deviation of 0.01 . Above this value, the target moves too fast for the robot to follow. 

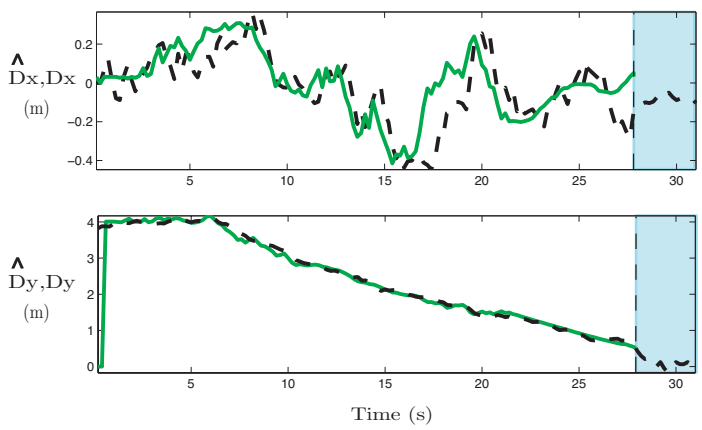

Fig. 6 Similar to fig. 4 but for the experiment 3.

\section{Conclusions}

The paper addresses the problem of generating timed trajectories for an autonomous robot that has to reach a location, within a specified time. While the robot is performing the task of reaching the target, it has to simultaneously compensate any delay or rush as a result of any obstacle circumnavigation or target displacements. The target (ball) is robustly detect by the robot's visual system through its color. Moreover, an EKF was developed and applied to estimate the location of the ball in cases where the visual system does not provide visual information.

We have successfully demonstrated the integration of a standard EKF and a nonlinear dynamical system to robotics in the same approach, without degrading the temporal properties of the proposed architecture. The inclusion of the EKF allowed the reduction of the error between the final position of the robot and that of the target.

Future work includes addressing more complex and cluttered environments, as well as to address sequentially tasks.

\section{Acknowledgement}

Work supported by the Portuguese Science Foundation (grant PTDC/EEA-CRO/100655/2008), and by project FCT PEst-OE/EEI/LA0009/2011. Jorge B. Silva is supported by PhD Grant SFRH/BD/68805/2010, granted by the Portuguese Science Foundation.

\section{References}

1. J. Silva, C. Santos, and V. Matos. Generating trajectories with temporal constraints for an autonomous robot. In 8th IEEE International Workshop on Safety, Security \& Rescue, Bremen, 
Germany, July 26-30, 2010.

2. J. Silva, C. Santos, and V. Matos. Timed trajectory generation for a toy-like wheeled robot. In 36th Annual Conference of the IEEE Industrial Electronics Society, Glendale, USA, November 07-10, pages $1645-1650,2010$.

3. A. Ijspeert, J. Nakanishi, and S. Schaal. Learning attractor landscapes for learning motor primitives. In Advances in Neural Information Processing Systems 15, pages 1547-1554. MIT Press, 2002.

4. C. Santos and V. Matos. Gait transition and modulation in a quadruped robot: A brainstem-like modulation approach. Robot. Auton. Syst., 59:620 - 634, 2011.

5. M. Buhler and S. Koditscheck. Planning and control of a juggling robot. International Journal of Robotics Research, 13(2):101 - 118, 1994.

6. E. Bizzi, P. Saltiel A. D'Avella, and M. Tresch. Modular organization of spinal motor systems. Neuroscientist, 8(5):437 - 442, 2002.

7. C. Maufroy, H. Kimura, and K. Takase. Towards a general neural controller for quadrupedal locomotion. Neural Networks, 21(4):667 - 681, 2008.

8. L. Righetti, J. Buchli, and A. J. Ijspeert. Dynamic hebbian learning in adaptive frequency oscillators. Physica D: Nonlinear Phenomena, 216(2):269 - 281, 2006.

9. C. Crick, M. Munz, and B. Scassellati. Synchronization in social tasks: Robotic drumming. In Proceedings of the 15th IEEE International Symposium on Robot and Human Interactive Communication, 2006, pages 97 - 102, 2006.

10. J. Kober and J. Peters. Learning motor primitives for robotics. In Proceedings of the IEEE International Conference on Robotics and Automation, Kobe International Conference Center, Kobe, Japan, pages $2112-2118,2009$.

11. M. Hersch and A.G. Billard. Reaching with multi-referential dynamical systems. Autonomous Robots, 25:71 - 83, 2008.

12. F. Stulp, E. Theodorou, M. Kalakrishnan, P. Pastor, L. Righetti, and S. Schaal. Learning motion primitive goals for robust manipulation. Proceedings of the IEEE International Conference on Intelligent Robots and Systems (IROS), pages 325 - 331, 2011.

13. M. R. Clark, G. T. Anderson, and R. D. Skinner. Coupled oscillator control of autonomous mobile robots. Autonomous Robots, 9:189 - 198, 2000.

14. G. Schöner. A dynamic theory of coordination of discrete movement. in Biological Cybernetics, 63:257-270, 1990.

15. C. Santos. Generating timed trajectories for an autonomous vehicle: a non-linear dynamical systems approach. in Proc. of the IEEE Int. Conf. on Robotics and Automation (ICRA), 2004.

16. M. Tuma, I. Iossifidis, and G. Schöner. Temporal stabilization of discrete movement in variable environments: an attractor dynamics approach. in IEEE International Conference on Robotics and Automation, Kobe International Conference Center, Kobe, Japan, pages 863 868, 2009.

17. S. Degallier and A. Ijspeert. Modeling discrete and rhythmic movements through motor primitives: A review. Biological Cybernetics, 103(4):319 - 338, 2010.

18. S. Schaal, S. Kotosaka, and D. Sternad. Nonlinear dynamical systems as movement primitives. International Conference on Humanoid Robotics, Cambridge, MA, pages 117 - 124, 2001.

19. A. Sekmen and B. Barshan. Estimaton of object location and radius of curvature using ultrasonic sonar. in IEEE Transactions on Pattern Analysis and Machine Intelligence, 62:841 $865,2001$.

20. C. Smith and H. I. Christensen. Using cots to construct a high performance robot arm. In Proceedings of the IEEE International Conference on Robotics and Automation, Roma, Italy, pages 4056 - 4063, 2007.

21. T. Wimböck B. Bäuml and G. Hirzinger. Kinematically optimal catching a flying ball with a hand-arm-system. In Proceedings of the IEEE/RSJ International Conference on Intelligent Robots and Systems (IROS), pages 2592 - 2599, 2010.

22. E. Bicho, P. Mallet, and G. Schöner. Target representation on an autonomous vehicle with low-level sensors. The International Journal of Robotics Research, (210):424-447, 2000. 